\title{
Effect of sputtering on self-damaged ITER-grade tungsten
}

\author{
V.S. Voitsenya ${ }^{\mathrm{a}}$, M. Balden ${ }^{\mathrm{b}}$, A.F. Bardamid ${ }^{\mathrm{c}}$, A.I. Belyaeva ${ }^{\mathrm{d}}$, V.N. Bondarenko ${ }^{\mathrm{a}}$, \\ O.O. Skoryk ${ }^{\mathrm{a}}$, A.F. Shtan’, S.I. Solodovchenko ${ }^{\mathrm{a}}$; V.A. Sterligov ${ }^{\mathrm{e}}$, B. Tyburska-Püschel ${ }^{\mathrm{b}}$ \\ ${ }^{a}$ Institute of Plasma Physics, National Scientific Center “'Kharkov Institute of Physics and Technology”, 61108 \\ Kharkov, Ukraine, \\ ${ }^{b}$ Institut für Plasmaphysik, 85748 Garching, Germany, \\ ${ }^{c}$ Taras Shevchenko National University, 01033 Kiev, Ukraine, \\ 'National Technical University "Kharkov Polytechnical Institute”, 61002 Kharkov, Ukraine, \\ ${ }^{e}$ Institute of Semiconductor Physics, NAS of Ukraine, 41 Nauki Prospect, 03028 Kiev, Ukraine
}

e-mail: voitseny@ipp.kharkov.ua

\begin{abstract}
Simulation of neutron irradiation and sputtering on ITER-grade tungsten was studied. The effects of neutron-induced displacement damage have been simulated by irradiation of tungsten target with $\mathrm{W}^{6+}$ ions of $20 \mathrm{MeV}$ energy. Bombardment by $\mathrm{Ar}^{+}$ions with energy $600 \mathrm{eV}$ was used as imitation of impact of charge exchange atoms in ITER. The sputtering process was interrupted to perform in between measurements of the optical properties of the eroded surface and the mass loss. After sputtering was finished, the surface was thoroughly investigated by different methods for characterising the surface relief developed due to sputtering. The damaging to, at least, the level that would be achieved in ITER does not lead to a decisive additional contribution to the processes under impact of charge exchange atoms only.
\end{abstract}

\section{Introduction}

It is known [1] that tungsten was chosen as the candidate material of the baffle and dome of the divertor, both will be plasma facing components of the vacuum vessel. Recently it was decided that divertor target plates will also be made of tungsten. According to [1], the baffle and divertor dome components with the total area about one hundred $\mathrm{m}^{2}$ will receive comparatively "low heat flux but high charge exchange neutral fluxes" of a wide energy distribution [2]. Because of divertor geometry the CXA (Charge Exchange Atoms) flux to divertor plates will probably be not critical to have a noticeable effect, but instead they will be undergone to repeated pulses of power plasma fluxes, e.g., by ELMs. Thus, the main criterion for the ITERgrade tungsten should be the high resistance to radiation damages due to impact of neutrons and CXA (D and T atoms) or neutrons and ions ( $\mathrm{D}^{+}, \mathrm{T}^{+}$and impurity ions). Simultaneous impact of both these factors can result in faster modification of the surface of tungsten in comparison with the case of CXA (or ions) only - due to the defects created by neutrons. Thus clearing-up this question is important for ITER with the present in-vessel construction conception.

For the tungsten to be used in ITER, so called, ITER-reference tungsten grade (ITER-grade $\mathrm{W})$, there are some requirements formulated in [3]. After analyzing properties of different kinds of W material the authors of [3] came to the conclusion: "Based on existing data base and results of R\&D, cost consideration and present design of the divertor components, the baseline choice of $\mathrm{W}$ grade is pure sintered tungsten (forged and/or swaged, cold or/and hot rolled and stress relieved)". And additionally: "To avoid loss of material due to delamination, the orientation of the texture has to be perpendicular to the surface of the W/Cu joints.”

At present a few companies produce tungsten with properties satisfying those requirements. The A.L.M.T. Corp. in Japan is one of these companies. Below we present and discuss the results obtained on ITER-grade W specimens, produced by A.L.M.T. Corp., after subjected to long-term sputtering with $\mathrm{Ar}^{+}$ions after irradiation with $20 \mathrm{MeV}$ ions $\mathrm{W}^{+6}$ (self-damaged ITERgrade tungsten). 
By this time several papers were devoted to simulate neutron irradiation effects by exposure of $\mathrm{W}$ specimens to $\mathrm{MeV}$ energy range $\mathrm{W}^{+6}$ ions (e.g., [4] and references herein) and its effect on the interaction of ITER-grade tungsten with deuterium. However, the important question relating the effects of neutron irradiation on formation of the surface relief under charge exchange atoms was only slightly touched in our previous paper [5]. In this paper we present results of detail investigations of surface structure of self-damaged ITER-grade tungsten specimens subjected to long-term sputtering.

\section{Experimental}

The experiments were performed on ITER-reference tungsten grade used previously for hydrogen retention studies [6]. The tungsten plate with purity of $99.99 \mathrm{wt} \%$ and with $99.7 \%$ of theoretical density was prepared (A.L.M.T. Corp., Japan) by a quite complicated technology [6]. Polycrystalline W workpieces were deformed (rolled, swaged and/or forged) with following appropriate heat-treatments to obtain better mechanical properties, e.g., strength and toughness. Then plates were cut into samples of $10 \times 10 \times 2 \mathrm{~mm}^{3}$, and double-side mechanically and electrochemically polished to a high optical mirror quality. Later in the text this type of tungsten is marked as WJ-IG.

The microstructure of the WJ-IG tungsten consists of anisotropically elongated grains along the deformation axis, and the grain size is around 1-3 $\mu \mathrm{m}$ in section and up to $5 \mu \mathrm{m}$ in length [6]. These grains are dominantly surrounded by small angle boundaries, which lead to a strong contrast in scanning electron microscopy (SEM). Large angle boundaries are obtained from electron backscatter diffraction (EBSD) data and the areas surrounded by these boundaries have sizes of $20-100 \mu \mathrm{m}$. The elongated grain orientation is defined to be parallel to the heat transfer direction, i.e., mainly perpendicular to the exposed surface, in accordance with the requirements [3].

To model neutron irradiation in ITER, two WJ-IG specimens were exposed to $\mathrm{W}^{+6}$ ions of $20 \mathrm{MeV}$ energy. The irradiated area (face side) was a circle with $8 \mathrm{~mm}$ in diameter. The calculated dose depth profile (shown in [7], Fig. 1) has a maximum (0.3 or $3.0 \mathrm{dpa}$ ) near $1.4 \mu \mathrm{m}$ and the maximal ion range was $\sim 2.2 \mu \mathrm{m}$; the details of calculations can be found in [8]. The rear side of specimens was not exposed to $\mathrm{W}^{+6}$ ions and used as the control surface in following ion sputtering procedures, simulating only CXA sputtering in ITER.

The initial reflectance of specimens was found to be noticeably below the nominal values for W [9], therefore a "soft" cleaning of their surfaces was provided in the DSM-2 device [10,11] before sputtering procedures were started. The rear sides (not irradiated with $\mathrm{W}^{+6}$ ions) were cleaned by exposing to low energy $(\sim 60 \mathrm{eV})$ ions of deuterium plasma (ion fluence $1.8 \times 10^{23} \mathrm{~m}^{-}$ ${ }^{2}$ ), and the face sides (irradiated) - by short time exposing to ions of Ar plasma with ion energy of $200 \mathrm{eV}$ (ion fluence $2 \times 10^{22} \mathrm{~m}^{-2}$ ). In both cases an electron cyclotron resonance (ECR) discharge (frequency $\mathrm{f}=2.35 \mathrm{GHz}$ ) was used for plasma production in deuterium and argon, respectively. Similar cleaning procedure is routinely used (e.g., [11]) for removing organic contaminants from the surface of mirror specimens prepared for the sputtering test. After cleaning, the reflectance of specimens became close to the data recommended in [9].

Following the cleaning process, a number of identical sputtering processes inside DSM-2 device were performed. The $\mathrm{Ar}^{+}$ions with ion energy of $600 \mathrm{eV}$ were used instead of hydrogen isotope ions to shorten the time of experiment (we suppose that relief due to sputtering does not depend on the mass of projectiles but on the thickness of sputtered layer only). The sputtering on both sides was performed in several steps, with the total accumulated ion fluence $6.5 \times 10^{23} \mathrm{ion} / \mathrm{m}^{2}$ for each side.

After each sputtering step the specimen surface was analyzed ex situ via optical microscopy, interferometry, reflectometry (measurement of reflectance at normal incidence $\mathrm{R}(\lambda)$ 
within $220 \div 650 \mathrm{~nm}$ ), and ellipsometry (ellipsometric parameters $\Psi$ and $\Delta$ within $450 \div 760 \mathrm{~nm}$ spectral range) dependent on Ar ion fluence. The results of optical and ellipsometrical measurements have been minutely described and discussed in the paper [12] and, therefore, they will be mentioned here only in the section "Discussion and Conclusions".

The mass loss was measured after each fluence step to obtain the sputtered layer thickness. After completing the sputtering, a layer of an average thickness of $3.9 \mu \mathrm{m}$ was eroded.

The resulting surfaces were analysed in detail with atomic force microscopy (AFM), confocal laser scanning microscopy (CLSM), and EBSD in a SEM.

Furthermore, the 2D spatial distribution of scattered light was measured using the installation described in [13]. In this installation incident laser light (He-Ne laser, $\lambda=632.8 \mathrm{~nm}$, $3 \mathrm{~mW}$ ) impinges onto the surface of an object along its normal. The object - W specimen - is placed in first focal point of an elliptical mirror. Light, scattered by this object, is directed by the elliptical mirror to its second focal point, where the lens of CCD camera is placed. Thus the whole half sphere distribution of scattered light intensity was registered. Scattered light intensity distribution $\mathrm{I}\left(\theta_{\mathrm{x}}, \theta_{\mathrm{y}}\right)$ measured along orthogonal polar angles $\theta_{\mathrm{x}}, \theta_{\mathrm{y}}$ is normalized to the incident light intensity $I_{0}$ and to the solid angle of each image pixel $d \Omega$ in order to calculate the quantity usually called Angle Resolved Scatter, ARS. It can be calculated as following:

$$
\operatorname{ARS}\left(\theta_{x}, \theta_{y}\right)=\frac{I\left(\theta_{x}, \theta_{y}\right)}{I_{0} \cdot d \Omega} .
$$

Obtained scattering data can also be well presented as a function of spatial frequency of the Fourier decomposition of a surface relief, which are inverse periods of diffraction gratings that are components of this relief decomposition:

$$
f=\frac{\sin \theta}{\lambda}=\frac{\sin \sqrt{\theta_{x}^{2}+\theta_{y}^{2}}}{\lambda} .
$$

\section{Experimental results}

\subsection{Investigation of surface structure}

\subsubsection{Interference microscopy, AFM, CLSM and EBSD data}

After cleaning, the surfaces of both sides were smooth as follows from the straight interference lines seen in an interferometer microscope [12]. However, with every sputtering step the interference patterns become more and more distorted and are practically indistinguishable between the last exposures to $\mathrm{Ar}^{+}$ions, Fig. 1(a). From this figure a preferred direction of elongated features is evident (marked by the black arrow). This preferential directional inhomogeneity of the relief is clearly seen also in scanning electron microscope (Fig. 1(b)) and atomic force microscopes (Fig. 2), as well as from CLSM and EBSD data (Figs. 3 and 4).

From EBSD data in Fig. 4 one can see that some areas with closely related colours are several tens of microns in size and have an obvious tendency to be extended about from top to bottom. The closely related colours mean that orientations of grains inside every of such area are close to each other. The analysis of the EBSD data regarding the misorientation between areas and applying a grain reconstruction after interpolation and spike removal in the data leads to areas surrounded by large angle boundaries $\left(>10^{\circ}\right)$ of dimensions of $20-100 \mu \mathrm{m}$. Correspondingly, the sputtering rate of each such area does not differ significantly. At the same time, difference between sputtering rates of conglomerates with other predominant grain orientations (other colours) can be very big. In agreement with the results of [7] the areas composed dominantly of grains with orientation close to (110) have highest sputtering rate, forming "valleys", and the areas composed dominantly of grains with orientation close to (100) 
have much lower sputtering rate forming the main parts of "ridges" with "peaks" composed of grains close to (111) orientation having lowest sputtering rate. Such surface relief is very much different from the relief of polycrystalline mirror specimens being sputtered under similar conditions $[\mathbf{7 , 1 0 , 1 1 , 1 4 ]}$, and this is a consequence of a quite complicated technology procedures used for preparation of this particular tungsten kind. Note that surface peculiarities due to sputtering are qualitatively similar for undamaged and damaged specimens, independently on the dose ( 0.3 or $3.0 \mathrm{dpa})$.

The structural data obtained in these experiments demonstrate that, similar to the cases of different polycrystalline metals [5], the real relief developing on the ITER-grade W surface under sputtering has much more large-scale characteristics than the micro-structural constituent elements of this tungsten kind, i.e., the size of crystallites of $\sim 5 \mu \mathrm{m}$ in depth and 1-3 $\mu \mathrm{m}$ [6] in width are not determinative for characteristics of microrelief that develops due to sputter erosion. This conclusion became a reason to apply the computer modelling of a relief (similar to [5]) with such strongly anisotropic structure, what is described in the next paragraph.

\subsubsection{Processing of CLSM data}

In order to determine the characteristics of the relief appeared on the specimen surface due to sputtering, the technique described in the Appendix of paper [5] was applied. Two basic distributions of inhomogeneity dimensions were calculated: (i) of longitudinal wavelengths formed by inhomogeneities along the surface, which can be named as "horizontal" distribution; (ii) of inhomogeneities in heights, named as "vertical" distribution.

The sampling was taken parallel to image edges of Fig. 3, i.e., approximately along the main direction of inhomogeneity (which does about coincide with the direction shown by the arrow in Fig. 1(a)) and at the angle $90^{\circ}$ to that direction. To provide a better statistics using the longest path for this procedure, the sampling was also taken along the rectangular spiral path spreading from the centre to the edges of the micrograph obtained by CLSM method for a surface fragment $1.1 \times 1.1 \mathrm{~mm}^{2}$. In such a way, the longitudinal wavelengths distribution $\Delta \mathrm{N} / \Delta \lambda$ was built using a number of longitudinal periods $\Delta \mathrm{N}$ per wavelength interval $\Delta \lambda$. The wavelengths were distributed among $\sim 300$ equal intervals ("bins") of width $\Delta \lambda \approx 5 \mu \mathrm{m}$. By analogy, the distribution $\Delta \mathrm{N} / \Delta \mathrm{h}$ of inhomogeneities in heights was calculated based on the number of heights $\Delta \mathrm{N}$ per height interval $\Delta \mathrm{h} \approx 0.04 \mu \mathrm{m}$, again by the use of the same CLSM data.

Fig. 5 shows the results ( 0 dpa specimen), for "horizontal" $\Delta N / \Delta \lambda$ and "vertical" $\Delta N / \Delta h$ distributions obtained with different sampling paths (results obtained for 0.3 dpa specimen were identical, thus are not shown). The mean value of "longitudinal wave", characterizing the distribution of inhomoheneities along the surface, $\Delta \mathrm{N} / \Delta \lambda$, equals to $\mathrm{S}_{\mathrm{m}}=38 \mu \mathrm{m}$, and is orders of magnitude larger than the typical grain size of this material $(1-3 \mu \mathrm{m})$. This is in agreement with the main result of [5]. Also the distribution $\Delta \mathrm{N} / \Delta \mathrm{h}$ is characterized by the root mean square deviation of the heights in a microrelief profile from the trend-line to that profile, $\mathrm{R}_{\mathrm{q}}=0.33 \mu \mathrm{m}$. It is seen that the maximal difference in height $(\sim 1.8 \mu \mathrm{m})$ is almost half of the mean thickness of sputtered layer $(\sim 3.9 \mu \mathrm{m})$. This points to the big difference in sputtering rate of faces (111) and (110) for $\mathrm{Ar}^{+}$ion bombardment with $600 \mathrm{eV} / \mathrm{Ar}$.

The results of the surface topography analysis obtained with straight line sampling (circles and crosses in Fig. 5(a)) do clearly indicate a strong anisotropy, in qualitative agreement with the data shown in other figures.

\subsection{Scattered light hemisphere distribution}

The sputtered surfaces of all samples are characterized by very high level of scattered light intensity. This is the consequence of a significant surface roughness developed due to sputtering. 
An example of the space distribution of the normalized scattered light intensity, ARS, for the sample damaged to 3 dpa is presented in Fig. 6. For the undamaged side the ARS distribution is similar, thus it was not shown. Significant azimuth anisotropy of scattered light intensity is evident from these data. This anisotropy is not related to the optical properties of installation, incident laser beam, or its polarization, but it is produced by optical properties of the sample itself. Azimuth sample anisotropy rotates scattering indicatrix by the same angle. Strong azimuth anisotropy of scattering distribution corresponds well with the azimuth anisotropy of microscopic surface image (Fig.1) and to data of other surface diagnostics (Figs. 2-4). This surface anisotropy is the origin for the azimuthal anisotropy of the measured indicatrix of scattered light presented in Fig. 6.

The result of the analysis of cross sections of scattering indicatrix along two different azimuth directions for face (damaged to $3.0 \mathrm{dpa}$ ) and undamaged sample side are presented in Fig. 7. Cross sections are made along orthogonal azimuth directions of the $\operatorname{ARS}\left(\theta_{\mathrm{x}}, \theta_{\mathrm{y}}\right)$ data, i.e., along $\mathrm{X}$ and $\mathrm{Y}$ axes of Fig. 6. They are characterized by biggest (Strong, corresponding to vertical azimuth direction) and smallest (Weak, corresponding to horizontal direction) scattering intensities. The presented results demonstrate that there are no significant differences in scattering properties of the damaged and undamaged side.

It is seen from Fig. 7 that scattering intensities of different cross sections are significantly different in a wide range of spatial frequencies. Such difference can be used to decrease the level of parasite scattered light by correct azimuth sample orientation in a way that direction of strong surface scattering will be normal to the plane of incidence, while direction of weak scattering will be parallel to the plane of incidence. The gain of such orientation can be numerically estimated according to the following relation:

$$
\operatorname{Gain}(\theta)=\frac{\operatorname{ARS}^{\text {Strong }}(\theta)}{A R S^{\text {Weak }}(\theta)},
$$

where $A R S^{\text {Strong }}(\theta), A R S^{\text {Weak }}(\theta)$ are ARS data taken along directions of strong and weak scattering correspondingly. The calculated $\operatorname{Gain}(\theta)$ data are presented in Fig. 8. From these data it is clear that in the $\theta$ range of $20^{\circ}-40^{\circ}$ parasite scattering can be decreased up to 5-6 times. The gain properties of the damaged and undamaged sample sides are rather similar.

\subsection{Weight loss}

The results of measurement of mass loss of WJ-IG specimens with different fluence of ions of argon plasma are presented in Fig. 9 for undamaged side and for the surface beforehand exposed to $\mathrm{W}^{+6}$ ions ( $3.0 \mathrm{dpa}$ ). As follows from these data, there is no significant difference between sputtering rate of specimens irradiated with $20 \mathrm{MeV} \mathrm{W}$ ions (filled circles) and the ones not irradiated (open squares), at least for sputtering with $\mathrm{Ar}^{+}$ions of an energy of $600 \mathrm{eV}$. (The average sputtering yield, 0.37 at/ion, is in a quite good agreement with the value $\sim 0.5$ at/ion indicated for $600 \mathrm{eV} \mathrm{Ar}+$ ions in the review paper [15].) The fact of independence of sputtering rate on whether the $\mathrm{W}$ specimen self-damaged or undamaged is important for ITER operation in the present version, where large surface area will be protected by ITER-grade tungsten.

\section{Discussion and conclusions}

The analysis of the experimental data shows a strong directional inhomogeneity of all measured parameters which appears due to sputtering independently, if the specimen was selfdamaged by $\mathrm{W}^{+6}$ ion irradiation or not. This directional inhomogeneity reflects the inhomogeneity of surface relief which develops with sputtering fluence. There is no doubt that the reason of relief inhomogeneity lies in the preparation technology of these particular W 
specimens leading to large areas of closely related orientation. The size of these areas exceeds (by more than one order of magnitude) the size of individual grains. The sputtering yield depends on the orientation [7]. Therefore, the obtained relief consisting of "ridges" and "valleys" corresponds to these areas of closely related orientation. They have an evident elongation in some preferred direction defined by the rolling plane (in Fig. 1 this direction is indicated by an arrow). According to Fig. 5(b), the height difference between lowest and highest levels reaches $\sim 1.8 \mu \mathrm{m}$ when the layer of $\sim 3.9 \mu \mathrm{m}$ was sputtered in average (weight loss measurements). In accordance with the EBSD results on the surface of recrystallized tungsten specimens [7], in "valleys" the grains with (110) orientation are predominant, and in "ridges" - the (100) grains with "peaks" corresponding to (111) orientations.

The independence of normal incidence reflectance degradation under long-term sputtering on whether $\mathrm{W}$ mirror specimens were pre-irradiated with $20 \mathrm{MeV} \mathrm{W}$ ions or not [12], is in agreement with previously published results on sputtering of copper and stainless steel mirror specimens pre-irradiated to varied doses with $\mathrm{Cu}$ and $\mathrm{Cr}$ ions of $3 \mathrm{MeV}$ energy, respectively [11], as well as with recently published data for recrystallized tungsten [7].

Rather intriguing are the results of ellipsometry, where a noticeable difference was found of parameters $\Psi$ and $\Delta$ for specimens irradiated ( $3 \mathrm{dpa}$ ) and non-irradiated with $20 \mathrm{MeV}$ W ions. The attempt to explain the difference of ellipsometry data with data of other methods was made in [12], where special investigation and modelling was provided based on comparative analysis of experimental data obtained by reflectometry and ellipsometry for specimens undamaged and self-damaged with $20 \mathrm{MeV} \mathrm{W}^{+6}$ ions.

The coarse-scaled roughness of the surface relief should strongly influence the reflection from tungsten surfaces of atoms and electromagnetic radiation emanating by burning plasma, and thus can change the heat balance of the baffle and dome elements of the divertor in ITER.

One manifestation of the surface relief inhomogeneity is a strong azimuth anisotropy of scattered light distribution, seen in Figs. 6-8. The directional inhomogeneity of the relief resulting in a factor of 5 to 6 difference in the scattered light intensity (Fig. 8) may be used for significant decrease of the stray light intensity in a given direction. This can help solving the stray light problem important for some optical diagnostics of plasma in ITER [16], if orientation of future inhomogeneity is known in advance, i.e., during installation of this $\mathrm{W}$ kind as protection tiles in ITER

Summarizing our results, we come to the conclusion important for exploitation of ITER: there should be no significant synergistic action of two key factors (neutrons and charge exchange atoms) on the sputtering rate and surface structure, at least, for the material of baffle and dome of the divertor, made of ITER-grade tungsten of similar kind.

\section{Acknowledgement}

The authors would like to thank M. Fußeder and J. Dorner for technical assistance for the $20 \mathrm{MeV} \mathrm{W}^{6+}$ irradiation and $\mathrm{V}$. Alimov for providing the $\mathrm{W}$ specimens and for useful discussion.

This project has received funding from the Euratom research and training programme 2014-2018. 


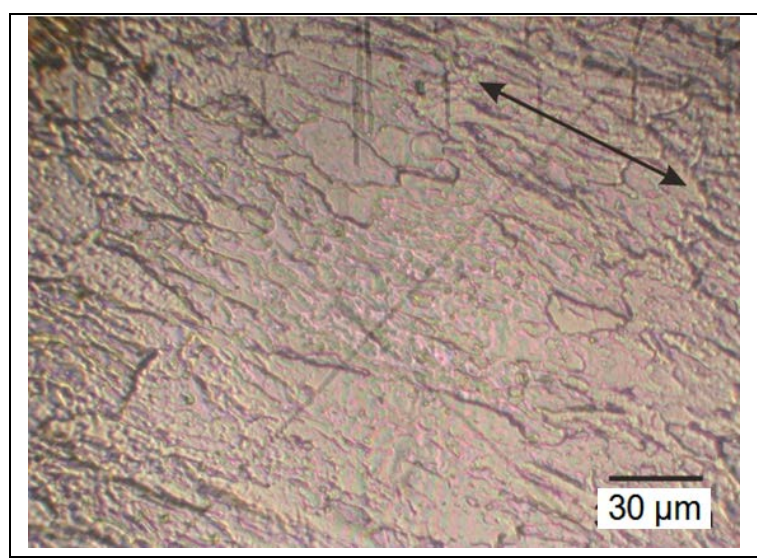

a)

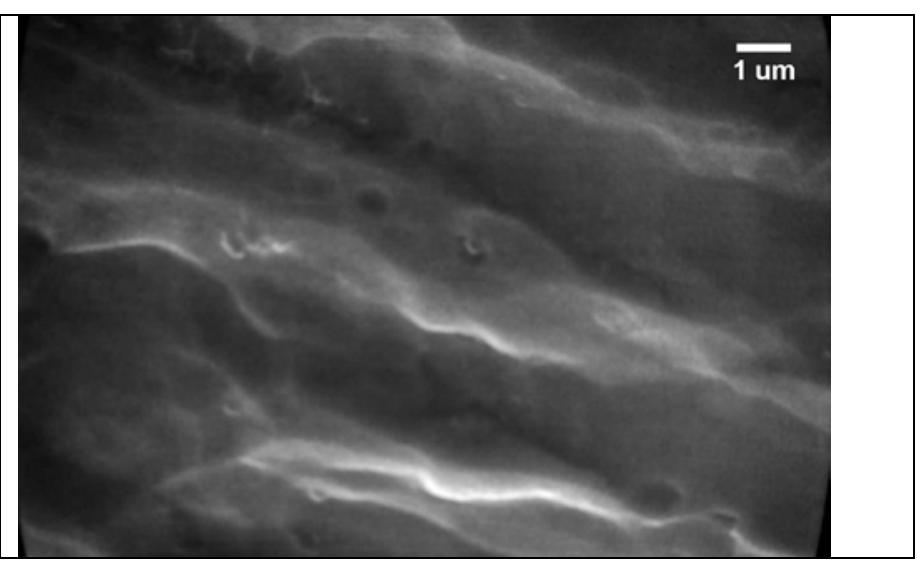

b)

Fig. 1(a) Photo from optical microscope and (b) SEM image of the WJ-IG (3.0 dpa) specimen after the layer of $\sim 3.9 \mu \mathrm{m}$ in thickness was sputtered (found by mass loss measurement).

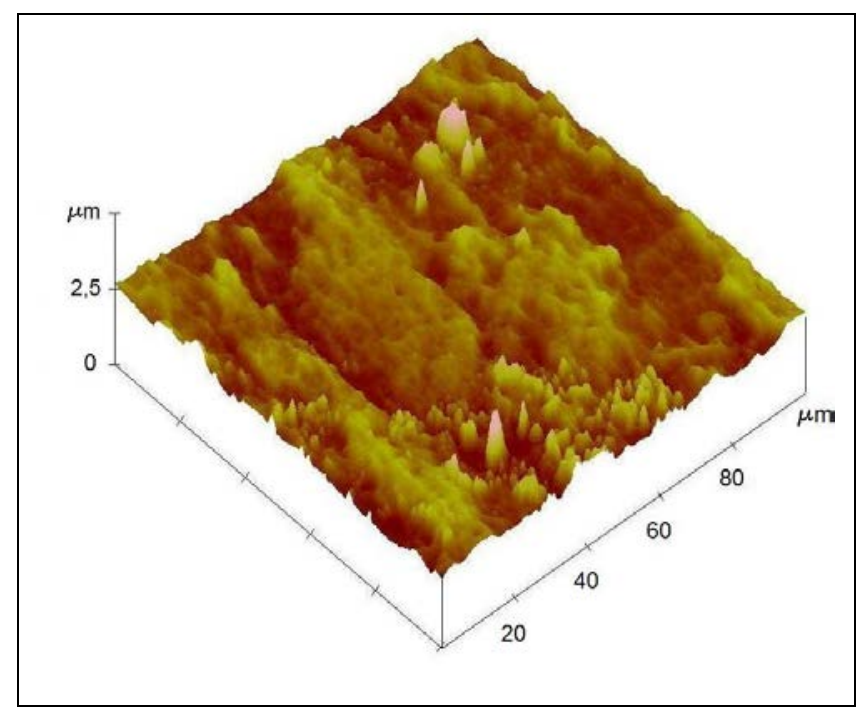

Fig. 2. AFM image of tungsten ITER-grade specimen (3.0 dpa) after sputtering a layer of $\sim 3.9 \mu \mathrm{m}$ in thickness with Ar ions from a ECR plasma. The imaged area is $100 \times 100 \mu \mathrm{m}^{2}$ and the height scale span $2.5 \mu \mathrm{m}$. 


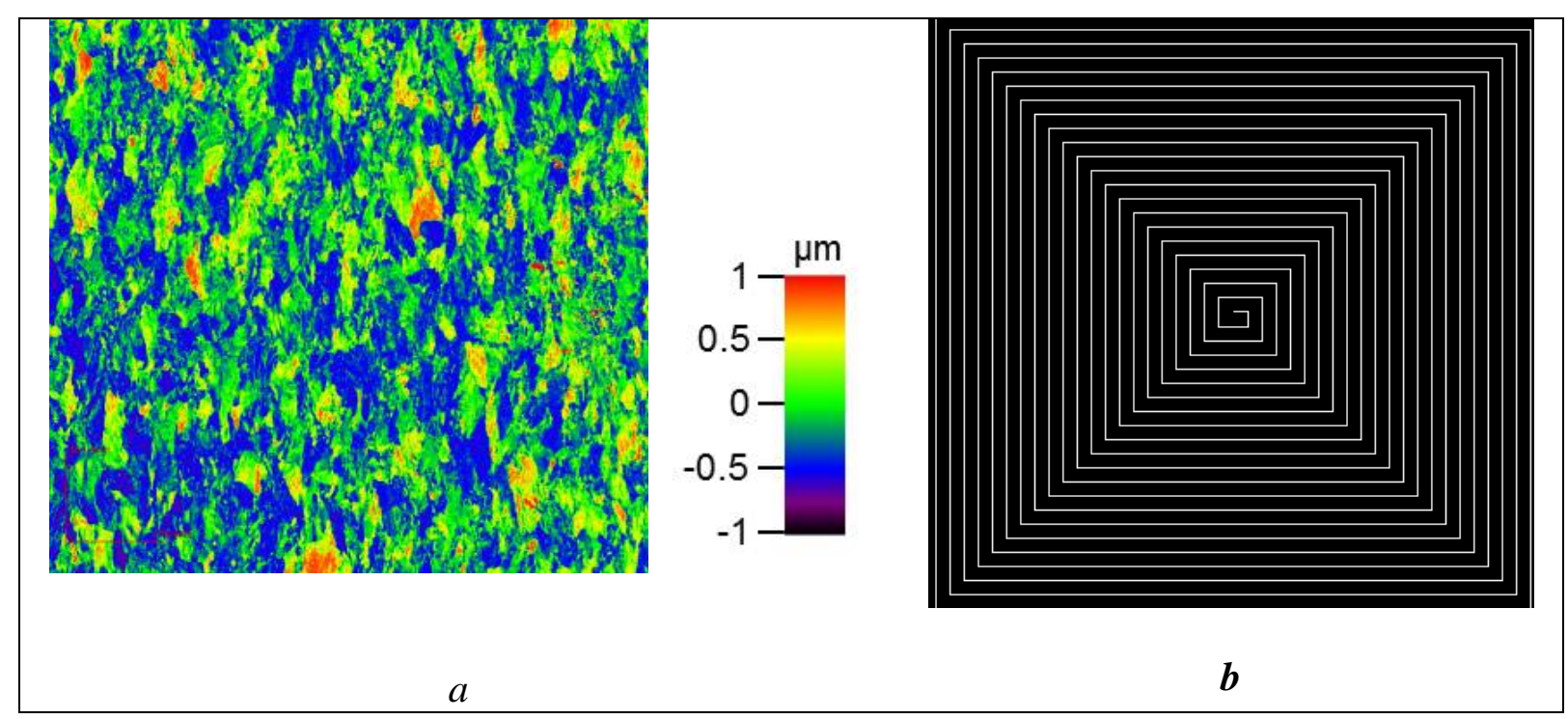

Fig. 3. a - height data of CLSM measurements for ITER-grade W ( $0.3 \mathrm{dpa})$. Size of the image is $1100 \times 1100 \mu \mathrm{m}^{2}$; b - near-central part of the rectanglural spiral (not in the scale with Fig.3a).

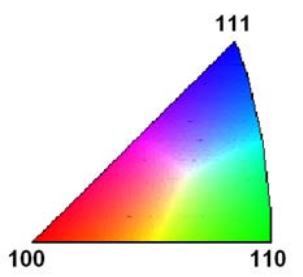

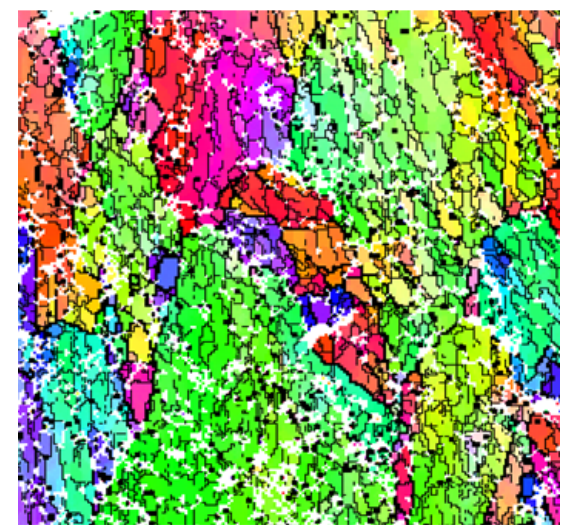

(a)

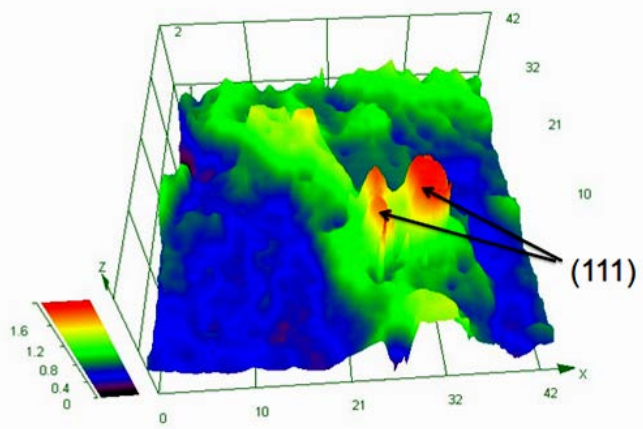

(b)

Fig. 4(a). EBSD data for Ar plasma sputtered $(\sim 3.9 \mu \mathrm{m})$ ITER-grade W specimen (0.3 dpa). Analysed area is $90 \times 90 \mu \mathrm{m}^{2}$. The color coding corresponds to the crystal planes orientated parallel to the surface. (b) 3D plot of the results of CLSM and EBSD measurements for small fragment $\left(43 \times 43 \mu \mathrm{m}^{2}\right)$ of the surface (shown in (a)). The height scale span $2 \mu \mathrm{m}$. 


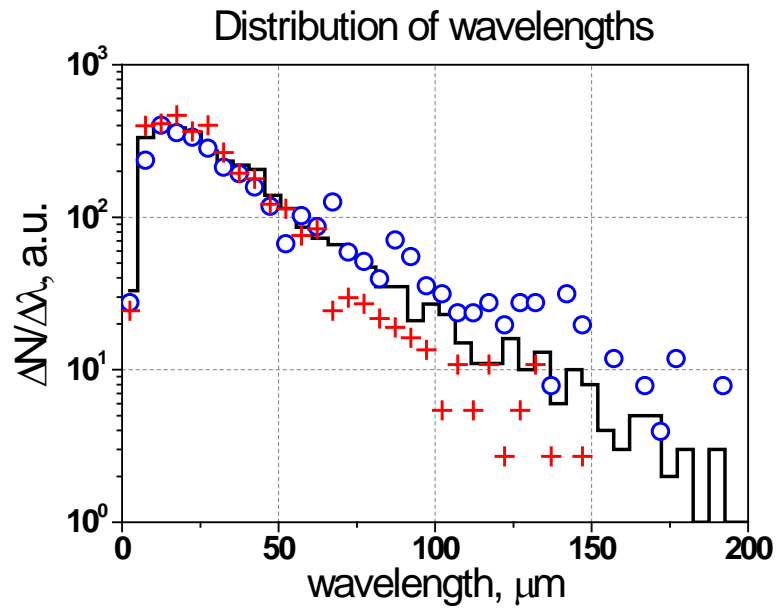

(a)

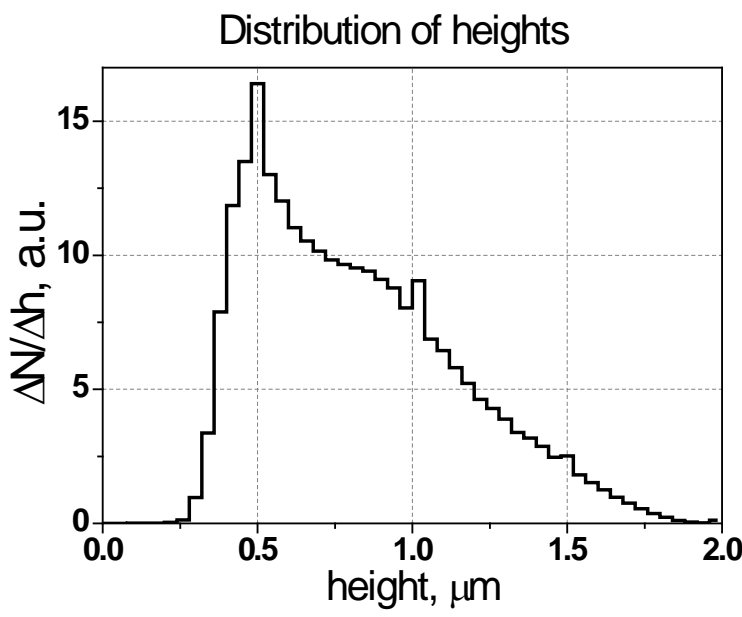

(b)

Fig. 5(a) Characteristics of inhomogeneities of surface relief along the surface for 0 dpa specimen when different paths for sampling of surface roughness characteristics were used: line - along a rectangular spiral, circles - parallel to the main direction of inhomogeneities (approximately parallel to the arrow in Fig. 1(a)), crosses - at the angle $90^{\circ}$ to the main direction. (b) vertical inhomogeneities of surface relief for the same specimen.

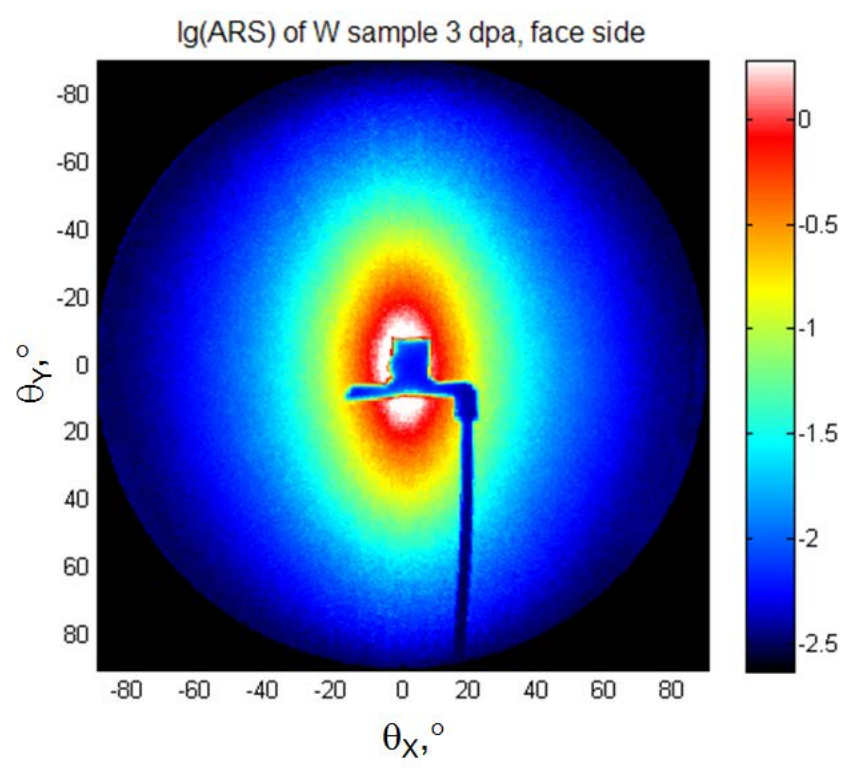

Fig. 6. Normalized distribution of scattering data for damaged side of a W sample (3 dpa). False colour coding of logarithm of ARS value is presented by colour bar. 


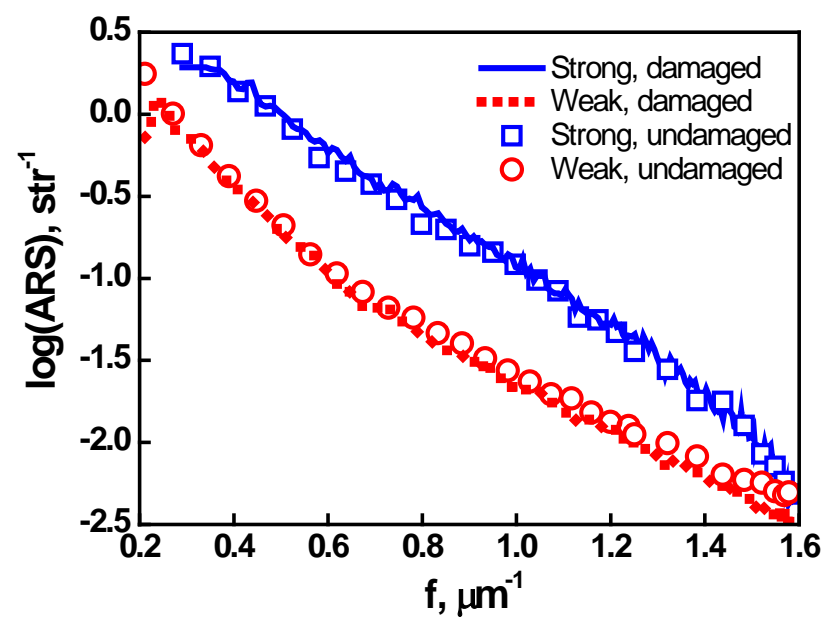

Fig. 7. Cross section of normalized scattering data for the 3 dpa damaged $\mathrm{W}$ sample as a function of spatial frequency of the surface relief decomposition. Scattering data for the face (damaged) side of the sample are solid lines, while the data for the rear (undamaged) side are solid lines with markers.

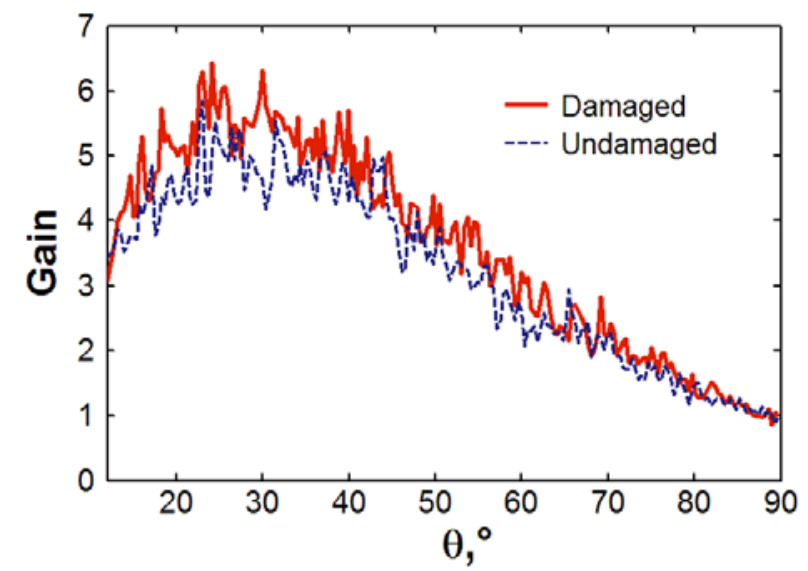

Fig. 8. Gain for the 3 dpa damaged W sample vs. polar angle of scattering $\theta$ for face (damaged) and rear (undamaged) sides.

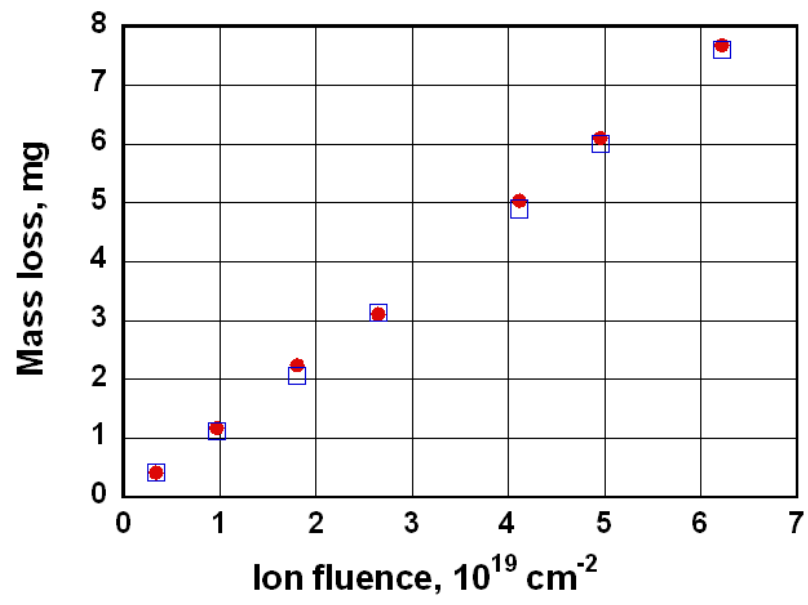

Fig. 9. Mass loss of WJ-IG specimens depending on the fluence of Ar plasma ions: open squares - not irradiated with $20 \mathrm{MeV}$ W ions (0 dpa); solid circles - pre-irradiated with $20 \mathrm{MeV}$ W ions (3.0 dpa). 


\section{References}

[1] G. Federici, C.H. Skinner, J.N. Brooks et al. Plasma material interactions in current tokamaks and their implications for next step fusion reactors. Nuclear Fusion 41, No. 12R (2001) 1967- 2137.

[2] R. Behrisch, G. Federichi, A. Kukushkin, et al. Material erosion at the vessel walls of future fusion devices. J. Nucl. Mater. 313-316 (2003) 388-392.

[3] Report G 74 MA 10 01-07-11 W 0.2, part 2.2 Tungsten.

[4] V.Kh. Alimov, Y. Hatano, B. Tyburska-Püschel, K. Sugiyama, I. Takagi, Y. Furuta, J. Dorner, M. Fußeder, K. Isobe, T. Yamanishi, M. Matsuyama. Deuterium retention in tungsten damaged with $\mathrm{W}$ ions to various damage levels. J. Nucl. Mater. 441 (2013) 280-285. .

[5] V.S. Voitsenya, M. Balden, A.F. Bardamid, V.N. Bondarenko, J.W. Davis, V.G. Konovalov, I.V. Ryzhkov, O.O. Skoryk, S.I. Solodovchenko, Zhou Zhang-jian. Development of surface relief on polycrystalline metals due to sputtering. Nucl. Instr. Methods Phys. Research B 302 (2013) 32-39.

[6] V.Kh. Alimov, B. Tyburska-Püschel, S. Lindig, Y. Hatano, M. Balden, J. Roth, K. Isobe, M. Matsuyama, T. Yamanishi. Temperature dependence of surface morphology and deuterium retention in polycrystalline ITER-grade tungsten exposed to low-energy, high-flux D plasma. J. Nucl. Mater. 420 (2012) 519-524.

[7] V.S. Voitsenya, M. Balden, A.I. Belyaeva, V.Kh. Alimov, B. Tyburska-Püschel, A.A. Galuza, A.A. Kasilov, I.V. Kolenov, V.G. Konovalov, O.O. Skoryk, S.I. Solodovchenko. Effect of sputtering on self-damaged recrystallized W mirror specimens. J. Nucl. Mater. 434 (2013) 375-381.

[8] B. Tyburska, V.Kh. Alimov, O.V. Ogorodnikova, K. Schmid, K. Ertl. Deuterium retention in self-damaged tungsten. J. Nucl. Mater. 395 (2009) 150-155.

[9] E.D. Palik (Editor), Handbook of Optical Constants of Solids (Academic Press, San Diego, California, 1985, 1991).

[10] A.F. Bardamid, V.T. Gritsyna, V.G. Konovalov et al. Ion energy distribution effect on degradation of optical properties of ion bombarded copper mirrors. Surf. Coatings Technol. 103-104 (1998) 365-369.

[11] D.V. Orlinski, V.S. Voitsenya, K.Yu. Vukolov. First mirrors for diagnostic systems of an experimental fusion reactor I. Simulation mirror tests under neutron and ion bombardment. Plasma Devices and Operations 15 (2007) 33-75.

[12] A.I. Belyaeva, A.A. Galuza, I.V. Kolenov, V.G. Konovalov, A.A. Savchenko, O.A. Skoryk, Effect of Sputtering on the Samples of ITER-Grade Tungsten Preliminarily Irradiated by Tungsten Ions: Optical Investigations //The Physics of Metals and Metallography 114 (2013) 703-713.

[13] V. Sterligov, P. Cheyssac. Appareil et procédé de caractérisation optique d'un objet; Patent CNRS (France): FR2832795, G01B-011/30; $\mathrm{n}^{\circ} 0115232$ of 23.11.2001]

http://worldwide.espacenet.com/publicationDetails/biblio?CC=FR\&NR=2832795

[14] M. Balden, A.F. Bardamid, A.I. Belyaeva, K.A. Slatin, J.W. Davis, A.A. Haasz, M. Poon, V.G. Konovalov, I.V. Ryzhkov, A.N. Shapoval. Surface roughening and grain orientation dependence of the erosion of polycrystalline stainless steel by hydrogen irradiation. J. Nucl. Mater. 329-333 (2004) 1515-1519.

[15] Ya. Yamamura and H. Tawara. Energy dependence of ion-induced sputtering yields from monatomic solids at normal incidence. Atomic Data and Nuclear Data Tables 62 (1996) 149-253.

[16] Shin Kajita, Evgeny Veshchev, Steve Lisgo, Roger Reichle, Robin Barnsley, Michael Walsh, Andrey Alekseev, Aleksey Gorshkov, Dmitry Vukolov, James Stuber and Simon Woodruff. Influence of stray light on visible spectroscopy for the scrape-off layer in ITER Plasma Phys. Control. Fusion 55 (2013) 085020 doi:10.1088/0741$\underline{3335 / 55 / 8 / 085020}$ 